
number and identity of deleterious variants in wild populations. For haplodiploid species, it has been missense and nonsense variants, than females from the same population. Allele frequency differences highly deleterious mutations are segregating in the paper wasp population. Stronger selection against

27 deleterious alleles in haploid males may have implications for adaptation in other haplodiploid insects 28 and provides evidence that wild populations harbor abundant deleterious variants.

\section{Sex differences in deleterious genetic variants in a haplodiploid social insect}

\author{
Sara E. Miller ${ }^{1 *}$, Michael J. Sheehan ${ }^{1 *}$
}

1. Department of Neurobiology and Behavior, Cornell University, Ithaca, NY

* Corresponding authors

\section{Abstract}

Deleterious variants are selected against but can linger in populations at low frequencies for long periods of time, decreasing fitness and contributing to disease burden in humans and other species.

Deleterious variants occur at low frequency but distinguishing deleterious variants from low frequency neutral variation is challenging based on population genetics data. As a result, we have little sense of the hypothesized that deleterious alleles will be directly exposed to selection in haploid males, but selection can be masked in diploid females due to partial or complete dominance, resulting in more efficient purging of deleterious mutations in males. Therefore, comparisons of the differences between haploid and diploid genomes from the same population may be a useful method for inferring rare deleterious variants. This study provides the first formal test of this hypothesis. Using wild populations of Northern paper wasps (Polistes fuscatus), we find that males have fewer overall variants, and specifically fewer are especially pronounced for rare missense and nonsense mutations and these differences lead to a lower genetic load in males than females. Based on these data we estimate that a large number of 


\section{Introduction}

Mutations are the raw source of variation for populations and most non-neutral mutations are

deleterious (1). Identifying deleterious and disease-causing variants and distinguishing these variants from

34 response of populations (2-4). Most deleterious variants are derived alleles present at low frequencies

35 within populations, however new neutral mutations share these same characteristics (5-7). As a result,

36 inferring the fitness effects of rare genetic variants from population genomics data remains a challenge,

37 especially for non-model organisms $(7,8)$.

In arrhenotokous haplodiploid species, males develop from unfertilized eggs as haploids whereas

females develop from fertilized diploid eggs. In essence, the entire haplodiploid genome is comparable to sex chromosomes in diploid species (9). This naturally occurring variation in ploidy between haplodiploid

41 sexes has led to a long-standing yet untested hypothesis that deleterious alleles will be directly exposed

42 to selection in males, but in females deleterious alleles can be masked from selection by partial or

43 complete dominance (10-12) (Figure 1). Thus, in haplodiploid species, selection against deleterious alleles

44 is predicted to occur more frequently in males than females, and is expected to lead to greater male

45 mortality as a consequence of the purging of lethal recessive mutations (13). Identifying which genetic

46 variants are differentially removed in haplodiploid males should therefore give insight into the

47 evolutionary dynamics of deleterious mutations.

In this study, we leveraged whole genome re-sequence data to directly test the hypothesis that

49 deleterious alleles are preferentially purged in haplodiploid males. There are several predictions following

50 from this hypothesis. First, samples of males should have fewer variants on average than samples of

51 females from the same population. However, a natural consequence of haplodiploidy is that at equal sex

52 ratios, females have a greater effective population size because females have double the number of copies

53 of each chromosome (12), and consequently, females are expected to have twice the effective mutation 
54 rate as males. Therefore, differences in the number of variants between sexes could also result from an

55 increased input of new mutations in females, rather than the removal of deleterious alleles in males.

56 Mutations are random throughout the genome; therefore, a second prediction of this hypothesis is that

57 if allele frequency differences between sexes are driven by selection, deleterious variants should be

58 underrepresented in males relative to females. Deleterious variants are those which reduce the fitness of

59 an organism. Across organisms, sites that modify protein amino acid sequences or influence transcription

60 are more conserved, indicating they are under stronger purifying selection. Therefore, we specifically

61 expect fewer nonsense or missense variants in males compared to females as these are most likely to

62 have negative fitness consequences. Finally, if deleterious variants are more often removed from the

63 male population, then strongly deleterious alleles will be absent or at a very low frequency in males but

64 may be present at a higher frequency in the female population. As a result, we would expect an overall

65 difference in allele frequencies between the sexes, and this difference should be most pronounced for

66 deleterious variants as neutral low frequency variants are predicted to have a similar frequency in both

67 males and females.

68 While there have been no direct tests of this hypothesis in haplodiploids, several lines of indirect

69 evidence support differences in selection rates between haploids and diploids. In plants, purifying

70 selection was greater on genes expressed only during the haploid phase than on genes expressed only

71 during the diploid phase (14). Haplodiploid insects have lower genetic variability (15) and reduced

72 inbreeding depression than diploid insects (16), consistent with haplodiploid species being more efficient

73 at purging deleterious mutations and thereby having a lower genetic load relative to diploid species (17).

74 Lastly, within haplodiploid species, if deleterious alleles are expressed more often in males, we might

75 predict that on average, males will be less capable of coping with agents of selection. Accordingly,

76 haplodiploid insect males have lower tolerance to pesticides than females (18), and male parasitoid wasps

77 have been shown to have higher mortality rates than females (13). 
We directly investigated this hypothesis using the Northern paper wasp, Polistes fuscatus, a

79 primitively eusocial arrhenotokous haplodiploid wasp with a widespread distribution across the Eastern

80 United States. In this species, reproductive males and reproductive females (gynes) are produced at an

81 approximately 1:1 ratio in the fall (19). Males disperse from their natal nests to nearby lekking sites to

82 mate, dying prior to winter. Gynes overwinter in hibernacula and start new nests the following spring (20,

83 21).

We collected 100 female and 185 male $P$. fuscatus from sites around Ithaca, New York from 2015-

2019. To account for possible differences in the per chromosome de novo mutation rate between sexes,

86 our dataset roughly balanced the number of chromosomes from males and females rather than the

87 number of individuals. Wasps from this region form a large single panmictic population with a high degree

88 of gene flow among sampling sites (22). All males and most females were reproductive individuals

89 collected in the fall (Table S1). Within each sex, individuals were unrelated, however males and females

90 from the same nest were included in the dataset. This heterogeneity in collection year and sampling site

91 within our dataset should, if anything, make it more challenging to detect systematic allele frequency

92 differences between the sexes.

Individually barcoded paired-end whole genome libraries were sequenced and aligned to the $P$.

94 fuscatus reference genome (23). The dataset was filtered to remove variants with low depth of coverage

95 to eliminate non-biological variants caused by sequencing errors as well as variants with unusually high

96 coverage to remove variants from unresolved duplicated regions caused by genome assembly errors. An

97 unavoidable consequence of haplodiploidy is that it is easier to detect true variants in males than females.

98 At the same sequencing depth, sequence data from females is split between the two copies of each

99 chromosome, while sequence data from males is from a single chromosome. Furthermore, heterozygous

100 genotypes in males are caused by sequencing errors, decreasing the rate of false positives in males.

101 Whereas females have a higher false negative rate due to an increased difficulty in distinguishing between 
sequencing errors and heterozygote genotypes, particularly at lower coverage sites. This difference in

103 variant detection error rates between males and females is expected to increase the number of high

104 confident variants in males relative to females, biasing the dataset towards a greater number of male

105 variants, which is in the opposite direction predicted by our hypothesis.

Females have more variants than males

We identified 4.6 million SNPs in the female samples and 3.6 million SNPs in male samples, matching our prediction that females will have more variants than males. However, due to the

110 heterogeneity in sample size and sampling regime between the sexes, it may be more meaningful to

111 compare the relative number of variants between the sexes rather than the total number of variants to

112 pinpoint which regions of the genome have stronger purifying selection in males. We calculated

113 nucleotide diversity $(\pi)$ and SNP density for males and females, plotted these values in 50,000 bp windows

114 across the genome, and calculated a z-score using the residuals between each point and the regression

115 line (Figure 2 ). Using a cutoff of $z>3$, there were 74 outlier windows ( $1.7 \%$ of windows) for $\pi$, and 30 outlier

116 windows ( $0.8 \%$ of windows) for SNP density. In all $\pi$ outlier windows, females had higher than expected

$117 \pi$. Similarly, 90\% of SNP density outlier windows had greater SNP density in females than males.

Variation in genetic diversity could conceivably be driven by sampling differences among male

119 and female samples rather than selective differences between the sexes. To test for the effect of sampling

120 error on estimates of $\pi$, we generated 100 random subsamples of females and males ( $n=100$

121 chromosomes/sex) and calculated the mean $\pi$ and mean SNP density of each replicate (Figure S1).

122 Consistent with the results from the full dataset, across all permutations, females had larger values of $\pi$

123 (Welch's t-test, $\left.t(163)=50, P<2.2 e^{-16}\right)$ and an increased number of SNPs (Welch's t-test, $t(197)=60, P<$

$\left.1242.2 \mathrm{e}^{-16}\right)$, indicating that sampling error had little effect on these results. 


\section{Males have fewer deleterious variants than females}

We investigated probable effects of SNPs and insertions/deletions (INDELs) variants in our dataset ratio could represent increased selection against transversions in the male population.

To test for the effect of sampling error, we applied a similar permutation approach as above,

(Welch's t-test, $\left.t(188)=171, P<2.2 e^{-16}\right)$, fewer nonsense variants (Welch's t-test, $t(116)=59, P<2.2 e^{-}$

${ }^{16}$ ), and a greater Ts/Tv ratio than females (Welch's t-test, t $(104)=-128, \mathrm{P}<2.2 \mathrm{e}^{-16}$ ) (Figure S2).

\section{Males have fewer deleterious variants in housekeeping genes}

If recessive deleterious alleles are preferentially exposed to selection in males, genes with

deleterious variants in females but not in males may be enriched for genes necessary for development or survival. We investigated the identity of genes with nonsense variants in one sex but not in the opposite

147 sex. We identified 773 genes with a nonsense variant in one or more of the female samples but without 148 a nonsense variant in males (i.e., conserved in males). In the opposite direction, we identified 317 genes 
were enriched for many gene ontology $(\mathrm{GO})$ terms associated with basic organismal processes necessary

151 for development such as mitotic spindle assembly checkpoint (GO:0007094), dorsal closure

152 (GO:0007391), regulation of cell fate specification (GO:0042659), and mushroom body development

153 (GO:0016319) (Table S2). Genes conserved in females were enriched for a wide range of GO terms

154 including regulation of female receptivity (GO:0045924) (Table S3).

155

\section{Males have fewer low frequency variants than females}

A third prediction of our hypothesis was that because most deleterious variants are likely at low

158 frequencies, increased selection against deleterious variants in males is expected to lead to a reduction in

the proportion of rare variants in males compared to females. To test this prediction, we first calculated

the number of single copy variants (singletons) in our male and female datasets. The female dataset had

$1611,529,219$ singletons whereas the male dataset had 1,309,484 singletons. Similar results were observed

162 when comparing subsets of males and females with an equal number of chromosomes (Figure S3).

We next compared the distribution of rare nonsense and missense alleles between the sexes

164 (Figure 3). For doubleton alleles (those with two copies in one of the sexes), we found that female 165 doubletons were more likely to be missing in males than male doubletons were missing in females, 166 illustrated by larger value of the red bar at a minor allele count of zero. Similar results were seen for alleles

167 with 3-5 copies. This finding suggests that males lack many of the rare deleterious alleles that are in the 168 female dataset. Synonymous variants in females showed a somwhat similar pattern as deleterious 169 variants (Figure S4), which could result from background selection against deleterious variants removing 170 linked synonymous variants.

171 Finally, considering the distribution of nonsense, missense, and synonymous variants across all 172 allele frequencies, we found that synonymous variants had a greater correlation between male and 173 female allele counts (Slope $\left.=0.88, R^{2}=0.95\right)$, than missense (Slope $=0.85, R^{2}=0.91$ ) or nonsense variants 
174 (Slope $\left.=0.70, R^{2}=0.75\right)$ (Figure S5). This weaker correlation between allele count at nonsense variants is

175 predicted if there is stronger selection against these deleterious alleles in males than females.

\section{Genetic load is lower in males}

178 Our previous analyses considered allele frequency differences between males and females at the 179 population level, but how does greater selection against deleterious alleles in the male population affect 180 the genetic load of individuals? We estimated genetic load by calculating the number of nonsense and 181 missense variants per individual. The average female is expected to have more variants than the average

182 male due to the extra copy of each chromosome, therefore we compared the number of deleterious 183 variants to the number of synonymous variants in each sample (Figure 4). The average male had $6.7 \%$ 184 fewer synonymous variants than the average female. However, this reduction was even more pronounced 185 for deleterious variants, with $17.7 \%$ fewer missense and $24.1 \%$ fewer nonsense variants in the average 186 male than the average female. Male samples had a lower genetic load than female samples, consistent 187 with our predictions.

\section{Discussion}

In haplodiploid species, selection against deleterious alleles has been predicted to occur more

191 frequently in haploid males than diploid females because recessive deleterious alleles in females can be 192 masked from selection by partial or complete dominance. Consistent with this theoretical prediction, we 193 found that haploid males have fewer overall variants, fewer deleterious variants, and fewer low frequency 194 deleterious variants than diploid females. There are challenges to directly comparing allele frequencies 195 between haploid and diploid groups due to differences in calling alleles for datasets with different ploidy, 196 but any such bias would not explain the reduction in low frequency deleterious variants in males relative 197 to females. 

between males and females that might explain our results. One possible mechanism might be a difference between male and female dispersal. A longer dispersal distance in one sex could increase the number of rare alleles detected in that sex. Prior comparisons of genetic diversity between the nuclear and

202 mitochondrial genome suggest that males may be dispersing somewhat further than reproductive 203 females (22). However, populations of $P$. fuscatus in Ithaca are panmictic (22) therefore gene flow from 204 nearby locations will likely have little effect on allele frequency differences between the sexes. Moreover, 205 longer-distance dispersal in males should increase the number of variants in males - the opposite effect 206 of what we observed in our study - making dispersal differences an unlikely explanation for our results. males and females. Although reproductive male and female $P$. fuscatus have a similar total number of 209 individuals (19), females have a larger effective population size than males because females have twice 210 the number of chromosomes. Natural selection is more efficient in larger populations, which could 211 potentially lead to more efficient purging of deleterious mutations in females. However, more effective 212 natural selection in females would lead to the inverse of our results, fewer genetic variants, and fewer 213 deleterious variants, in females compared to males. Effective population size differences can also affect 214 the number of new mutations contributed by each sex. With an equal mutation rate, new mutations will 215 arise more often in diploid females than haploid males. Biases in certain nucleotide substitutions have 216 been detected between haploid and diploid yeast (26) and an elevated mutation rate in females could

217 also increase the number of rare alleles in females. However, in either case, the effect of increased 218 mutational input from females will be short-lived. All mutations originating in females will be expressed 219 in the next generation of males. The precise dynamics of the spread of new mutations in $P$. fuscatus 220 depend on the mutation-selection balance in this species, which will require future study, but it seems 221 unlikely that mutation rate differences alone are the primary cause of our results. 
Another source of allele frequency variation between the sexes is sexually antagonistic selection

223 (SAS), in which an allele is beneficial to one sex and harmful to the other sex $(27,28)$. It has been theorized

224 that haplodiploids might be predisposed to extensive SAS and intralocus sexual conflicts in haplodiploids

225 are predicted to be resolved in favor of females (29). In the tawny crazy ant (Nylanderia fulva), SAS for

226 both male and female biased alleles was detected for $\sim 3 \%$ of the genome (30). However, SAS has not been

227 extensively investigated in haplodiploids and it is unknown how widespread SAS is across haplodiploid

228 species $(27,28)$. While SAS may explain some of the allele frequency differences between the sexes, it

229 does not explain why allele frequency differences between the sexes are greatest for low frequency

230 nonsense and missense variants.

The most likely and parsimonious explanation for our results is stronger purifying selection against deleterious mutations in haploid males. The magnitude of allele frequency differences between males

233 and females at some sites in our dataset was quite large. In the most extreme case of selection, recessive

234 lethal mutations would cause male mortality during development (Figure 1). Developing and dead larvae

235 have been observed to be regularly ejected from nests (31), in one reported case, $58 \%$ of larvae were

236 removed from a P. dominula nest (Pardi 1951 cited in 31). Researchers have not investigated the sex of

237 removed larvae, but intriguingly larvae were rejected more frequently in the fall, which is when males are

238 produced (31). Additionally, neutral, or deleterious variants linked to developmentally lethal alleles will

239 also be removed from the male gene pool via background selection further decreasing genetic variation

240 in the male population. Consequently, sisters may carry haplotypes, or even large chromosomal regions

241 that are absent in their brothers.

Moderately or weakly deleterious alleles can impact male survival at later life stages, thereby

243 further removing additional genetic variation from the sampled male population. Male $P$. fuscatus gather

244 at leks or defend small territories near females (32), and many of our male specimens were collected at 245 lekking sites. Males with a greater genetic load may be of poorer quality and therefore less capable of 
survival and competition, leading to earlier mortality, fewer mating opportunities, and stronger selection

247 against males with deleterious alleles.

Haplodiploidy is a widespread method of sex determination, occurring in nearly $15 \%$ of arthropod species, with at least 14 independent origins of arrhenotokous haplodiploidy in insects (33, 34). If purifying selection is indeed stronger against haploid males than diploid females, this has several interesting

251 implications for adaptation in haplodiploids. For example, the ant species Formica aquilonia and Formica

252 polyctena hybridize in nature producing viable female offspring and inviable male offspring $(36,37)$, likely

253 as a result of incompatibilities between recessive alleles in the two parental species. Selection against

254 deleterious recessive alleles in haploid males is predicted to lower the genetic load and reduce inbreeding

255 depression in haplodiploid species compared to diploid species $(16,17)$. This reduced genetic load may

256 facilitate the development of mating systems with high levels of inbreeding such as the bark beetles (35).

257 However, lower levels of genetic diversity in haplodiploid species could potentially slow rates of molecular

258 evolution in comparison to diploid species. Alternatively, haplodiploids could compensate for lower

259 genetic diversity through alternative mechanisms such as increased dispersal, or higher mutation or

260 recombination rates. Indeed, comparisons among a limited number of species have found higher

261 recombination rates in haplodiploid species compared to other insect species (38). Within haplodiploid

262 species, haploid males may be more sensitive than females to the effects of environmental change,

263 pesticide use, or disease. Future studies of environmental perturbations on haplodiploid insects may want

264 to consider the effects separately between males and females. Comparisons between genetic variants

265 present in diploid females but missing in haploid males has potential for rapidly identifying putative

266 deleterious variants in natural populations. 
271 around Ithaca, NY from 2015-2019 (Table S1). There is a high degree of gene flow across this region, with

272 P. fuscatus forming a single panmictic population (22). Our dataset included all unrelated males collected

$273(\mathrm{~N}=185)$. Female samples $(\mathrm{N}=100)$ were selected from a larger dataset of sequenced females from the

274 Ithaca region. We chose female samples collected from similar collection sites as the male dataset,

275 excluding females with kinship coefficients $>0.1$ as identified with the -relatedness 2 option in VCFtools

276 (v0.1.15) (39), and retaining samples with the highest depth of sequencing coverage. In some cases, males

277 and females collected from the same nest were included in the dataset (Table S1).

278 DNA was extracted from wasp legs with the Qiagen Puregene Core Kit A. Individually barcoded

279 paired-end whole-genome libraries were constructed with the Nextera library preparation kit with an 280 average insert size of $550 \mathrm{bp}$. Library were sequenced on the Illumina HiSeq 2000 and NovaSeq 6000.

Multiple filters were applied to sequenced data to remove variants caused by sequencing error

284 rather than true biological variation. Raw sequencing reads were processed with Trimmomatic (v0.36) to

remove adaptors and poor-quality sequence using the options SLIDINGWINDOW:4:15, LEADING:3,

TRAILING:3, and MINLEN:36. Trimmed reads were aligned to the $P$. fuscatus reference genome (23) using

287 the Burrows-Wheeler Aligner (v0.7.13) (40). Due to downstream software issues with having haploid and 288 diploid variants in the same VCF file, variants were called separately in males and females. Variants were 289 identified using Picard tools (v2.8.2; http://broadinstitute.github.io/picard) and the HaplotypeCaller tool 290 in GATK (v3.8) (41). Variant calls for males and females were merged separately with the GenotypeGVCF 291 tool in GATK, which aggregates variants across samples to correct genotype likelihoods and improve the 292 confidence of variant identification. After alignment, poor confidence variants were hard filtered 293 following GATK best practices recommendations. Single nucleotide polymorphisms (SNPs) were filtered 
using the parameters QUAL $<30$, SOR $>3.0, \mathrm{FS}>60, \mathrm{MQ}<40$, MQRankSum $<-12.5$, and ReadPosRankSum

$<-8.0$. Insertion/Deletion (INDEL) variants were filtered using the parameters $\mathrm{QD}<2.0, \mathrm{QUAL}<30, \mathrm{FS}>$ removing variants with more than $80 \%$ missing data, and variants with exceptionally low or high depth of sequencing coverage across all individuals, using the filters --min-meanDP 3, --max-meanDP 500, and -max-missing 0.2 in VCFtools (39).

Measurement of genetic variation across the genome and mean SNP density in 50,000 bp window using VCFtools (39). We plotted the mean value of $\pi$ or SNP

305 density for each sex, and then calculated a z-score for each window using the distance from each point to 306 the regression line. Windows with z-scores $>3$ or $<-3$ were considered outlier windows. Postitive z-scores 307 indicate that $\pi$ or SNP density was higher in females then expected. of chromosomes between the sexes) and calculated the mean genome-wide $\pi$ and SNP density. We

311 repeated this for 100 random subsets of males and females. Differences between the male and female 312 subsamples were assessed using t-tests implemented in R (v4.0.2) (42). 
317 To assess the possible effect of sampling error on these results, we repeated SnpEff on the same 100

318 subsets of 100 females or 50 males in the permutation test described above.

319 Using the output of SnpEff, we assembled a list of genes containing at least one nonsense variant

320 in one or more of the females in our dataset but lacking nonsense variants in the male dataset ("conserved

321 in males"). For genes conserved in males, we looked for enrichment of Gene Ontology (GO) terms using

322 the TopGO package in R (43), choosing the 'weight01' algorithm to incorporate the GO hierarchy when

323 identifying enriched GO terms, and assessing significance using fisher's exact test. We repeated this

324 analysis for genes without nonsense variants in females but with at least one nonsense variant in our male

325 dataset ("conserved in females").

326 To estimate genetic load of each sample, we used SnpEff to identify the number of missense,

327 nonsense, and synonymous variants in each individual sample.

We used VCFtools (39) to calculate the number of single copy alleles in the complete male and

331 female datasets and in the 100 subsets of 100 females or 50 males. We then compared the distribution

332 of nonsense, missense, and synonymous alleles between the male and female datasets. We compared

333 the number of alleles rather than the allele frequency to avoid inflating allele frequencies for sites with

334 lower coverage across samples. Variants were filtered by effect using SnpSift (v4.3) (24) and the minor

335 allele count was calculated at each site using VCFtools (39). As the original VCF file contained only variable

336 sites, variants that were missing in the opposite sex could be caused by lack of sequence data at that site,

337 or because that site is invariant in the opposite sex. To distinguish between these possibilities, we output

338 all invariant sites in the male and female dataset using GATK and kept only sites that were present in both

339 sexes. 


\section{References}

342 1. A. Eyre-Walker, P. D. Keightley, The distribution of fitness effects of new mutations. Nat. Rev. Genet. 8, 610-618 (2007).

344 2. G. M. Cooper, J. Shendure, Needles in stacks of needles: Finding disease-causal variants in a wealth of genomic data. Nat. Rev. Genet. 12, 628-640 (2011).

3. S. Chun, J. C. Fay, Identification of deleterious mutations within three human genomes. Genome Res. 19, 1553-1561 (2009).

4. D. A. Bell, et al., The Exciting Potential and Remaining Uncertainties of Genetic Rescue. Trends Ecol. Evol. 34, 1070-1079 (2019).

5. Q. Zhu, et al., A genome-wide comparison of the functional properties of rare and common genetic variants in humans. Am. J. Hum. Genet. 88, 458-468 (2011).

6. G. Gibson, Rare and common variants: Twenty arguments. Nat. Rev. Genet. 13, 135-145 (2012).

7. B. Y. Kim, C. D. Huber, K. E. Lohmueller, Inference of the distribution of selection coefficients for

8. P. Tataru, M. Mollion, S. Glémin, T. Bataillon, Inference of distribution of fitness effects and proportion of adaptive substitutions from polymorphism data. Genetics 207, 1103-1119 (2017).

9. S. Immler, Haploid Selection in "diploid" Organisms. Annu. Rev. Ecol. Evol. Syst. 50, 219-236 (2019).

10. D. L. Hartl, Some aspects of natural selection in arrhenotokous populations. Integr. Comp. Biol.

11. J. H. Werren, "The evolution of inbreeding in haplodiploid organisms" in The Natural History of

12. P. W. Hedrick, J. D. Parker, Evolutionary genetics and genetic variation of haplodiploids and X-

13. R. H. Smith, M. R. Shaw, Haplodiploid sex ratios and the mutation rate. Nature 287, 728-729 (1980).

14. R. Arunkumar, E. B. Josephs, R. J. Williamson, S. I. Wright, Pollen-specific, but not sperm-specific, genes show stronger purifying selection and higher rates of positive selection than sporophytic genes in capsella grandiflora. Mol. Biol. Evol. 30, 2475-2486 (2013).

15. B. Y. P. J. Avery, The population genetics of haplo-diploids and X-linked genes. Genet. Res.

16. H. J. Henter, Inbreeding depression and haplodiploidy: Experimental measures in a parasitoid and

17. S. P. Otto, M. F. Scott, S. Immler, Evolution of haploid selection in predominantly diploid

18. Y. Carrière, Haplodiploidy, Sex, and the Evolution of Pesticide Resistance. J. Econ. Entomol. 96,

19. K. M. Noonan, Sex ratio of parental investment in colonies of the social wasp Polistes fuscatus. Science (80-. ). 199, 1354-1356 (1978).

20. H. K. Reeve, "Polistes" in The Social Biology of Wasps, (1991). 
21. S. E. Miller, et al., WASPnest: a worldwide assessment of social Polistine nesting behavior. Ecology 99, 2405 (2018).

22. S. E. Bluher, S. E. Miller, M. J. Sheehan, Fine-scale population structure but limited genetic differentiation in a cooperatively breeding paper wasp. Genome Biol. Evol. (2020) https:/doi.org/10.1093/gbe/evaa070.

23. S. E. Miller, et al., Evolutionary dynamics of recent selection on cognitive abilities. Proc. Natl. Acad. Sci. U. S. A. 117, 3045-3052 (2020).

24. P. Cingolani, et al., A program for annotating and predicting the effects of single nucleotide

25. D. M. Lyons, A. S. Lauring, Evidence for the selective basis of transition-to-transversion

26. N. P. Sharp, L. Sandell, C. G. James, S. P. Otto, The genome-wide rate and spectrum of

27. R. M. Cox, R. Calsbeek, Sexually antagonistic selection, sexual dimorphism, and the resolution of spontaneous mutations differ between haploid and diploid yeast. Proc. Natl. Acad. Sci. U. S. A. 115, E5046-E5055 (2018).

intralocus sexual conflict. Am. Nat. 173, 176-187 (2009).

28. C. Cheng, M. Kirkpatrick, Sex-Specific Selection and Sex-Biased Gene Expression in Humans and Flies. PLoS Genet. 12, 1-18 (2016).

29. K. Kraaijeveld, Male genes with nowhere to hide; Sexual conflict in haplodiploids. Anim. Biol. 59, 403-415 (2009).

30. P. A. Eyer, A. J. Blumenfeld, E. L. Vargo, Sexually antagonistic selection promotes genetic divergence between males and females in an ant. Proc. Natl. Acad. Sci. U. S. A. 116, 24157-24163 (2019).

31. S. C. R. Turillazzi, "Oophagy and infanticide in colonies of social wasps" in Infanticide and Parental

32. D. C. Post, R. L. Jeanne, Male Reproductive Behavior of the Social Wasp Polistes fascatus Care, (Harwood Academic Publishers, 1994), pp. 213-236.

(Hymenoptera: Vespidae). Z. Tierpsychol. 62, 157-171 (1983).

33. B. B. Normark, The Evolution of Alternative Genetic Systems in Insects. Annu. Rev. Entomol. 48, 397-423 (2003).

34. A. G. De La Filia, S. A. Bain, L. Ross, Haplodiploidy and the reproductive ecology of Arthropods. Curr. Opin. Insect Sci. 9, 36-43 (2015).

35. L. Keller, K. Peer, C. Bernasconi, M. Taborsky, D. M. Shuker, Inbreeding and selection on sex ratio in the bark beetle Xylosandrus germanus. BMC Evol. Biol. 11 (2011).

36. J. Kulmuni, B. Seifert, P. Pamilo, Segregation distortion causes large-scale differences between male and female genomes in hybrid ants. Proc. Natl. Acad. Sci. U. S. A. 107, 7371-7376 (2010).

37. J. Kulmuni, P. Pamilo, Introgression in hybrid ants is favored in females but selected against in males. Proc. Natl. Acad. Sci. U. S. A. 111, 12805-12810 (2014).

38. L. Wilfert, J. Gadau, P. Schmid-Hempel, Variation in genomic recombination rates among animal taxa and the case of social insects. Heredity (Edinb). 98, 189-197 (2007).

421 40. H. Li, R. Durbin, Fast and accurate long-read alignment with Burrows-Wheeler transform. 
Bioinformatics 26, 589-595 (2010).

423

41. G. A. Van der Auwera, et al., From fastQ data to high-confidence variant calls: The genome analysis toolkit best practices pipeline. Curr. Protoc. Bioinforma. (2013) https:/doi.org/10.1002/0471250953.bi1110s43.

42. R Core Development Team, R: A language and environment for statistical computing. Vienna, Austria (2019).

43. topGO: Enrichment analysis for Gene Ontology. R Packag. version 2.22.0. Bioconductor. 


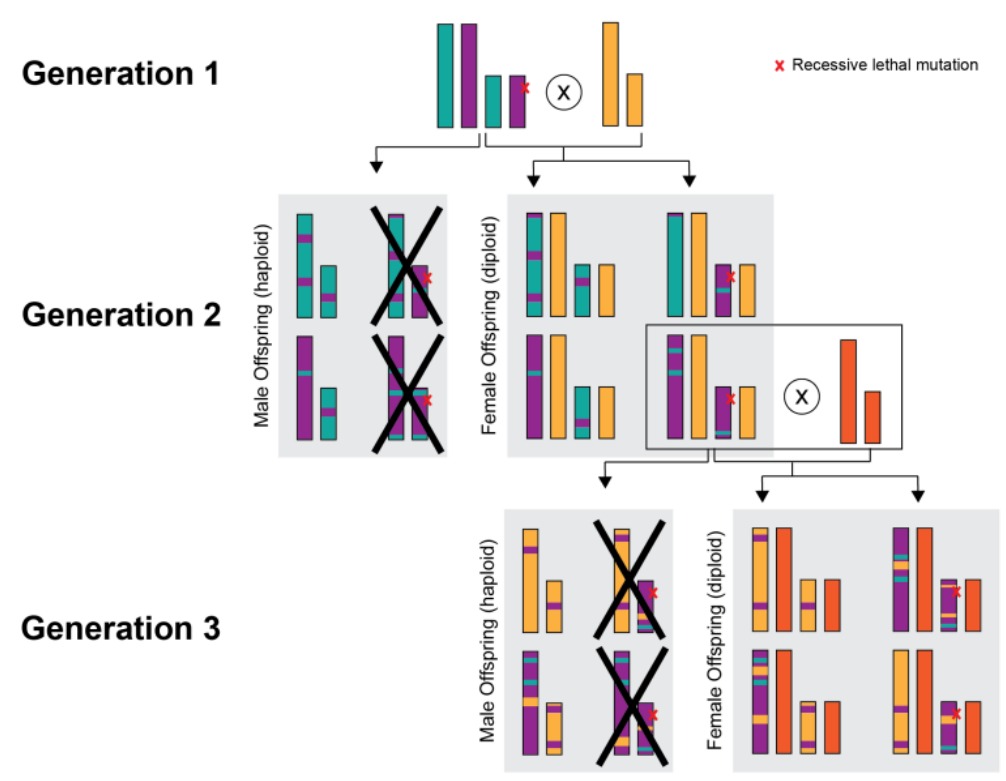

431 Figure 1: Schematic of differential selection against a recessive lethal mutation in an arrhenotokous 432 haplodiploid species $(\mathrm{N}=2)$. All males with the recessive lethal mutation die but females must have two 433 copies of the allele to be affected. After three generations, fifty percent of male offspring have been 434 removed from the male gene pool while there was no selection against female offspring with the allele. 435 No recombination occurs in haploid males. 

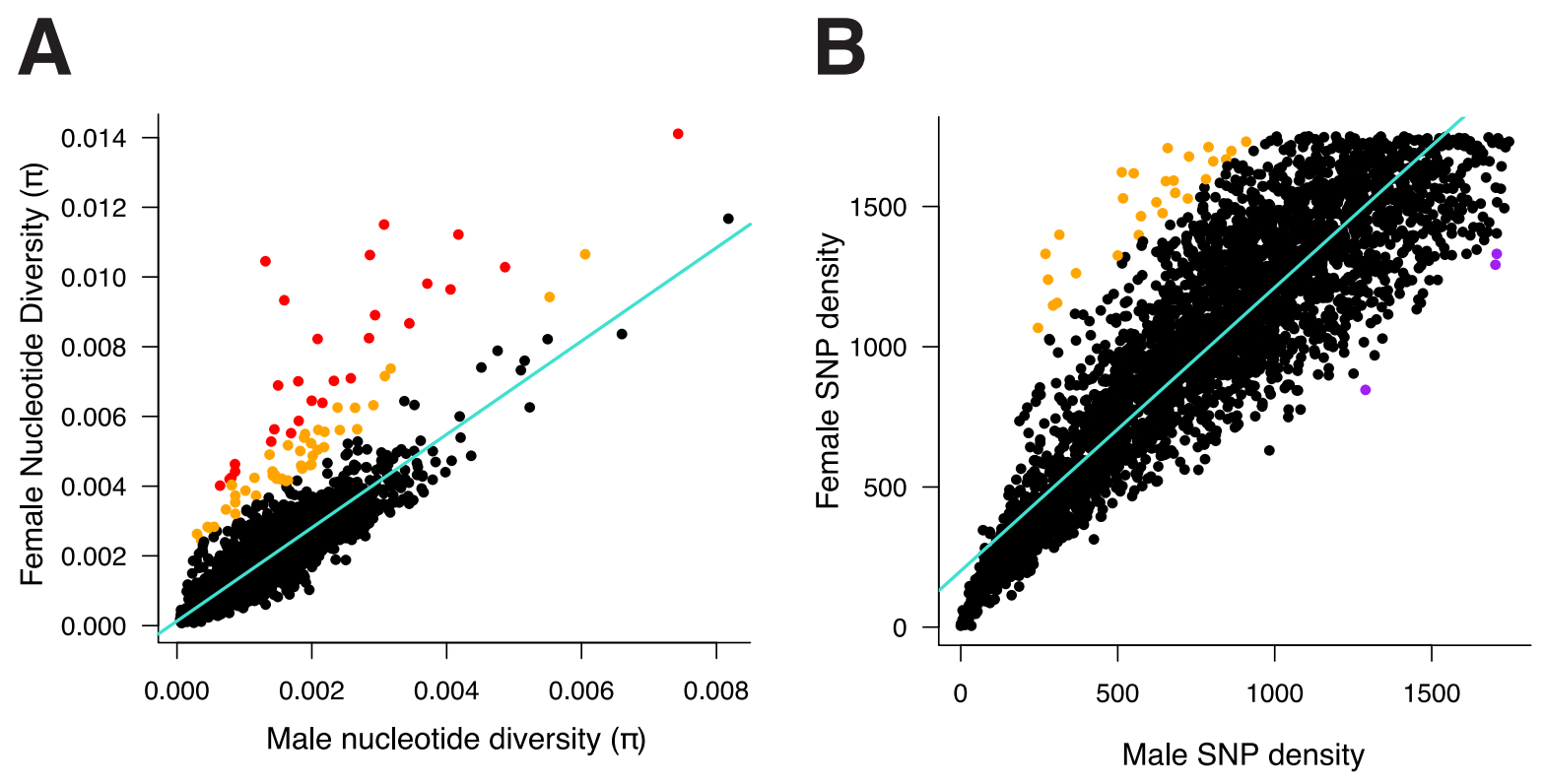

436

Figure 2: Comparison of genetic variation between females and males. (A) Nucleotide diversity $(\pi)$ and $(B)$ SNP density for males and females. Each point show the mean value of $\pi$ or SNP density within a 50,000 bp window. A z-score was calculated using the distance from each point to the regression line (shown in teal). Points with z-scores $>3$ are highlighted in orange, z-scores $>5$ in red, and points with z-scores $<-3$ are highlighted in purple 

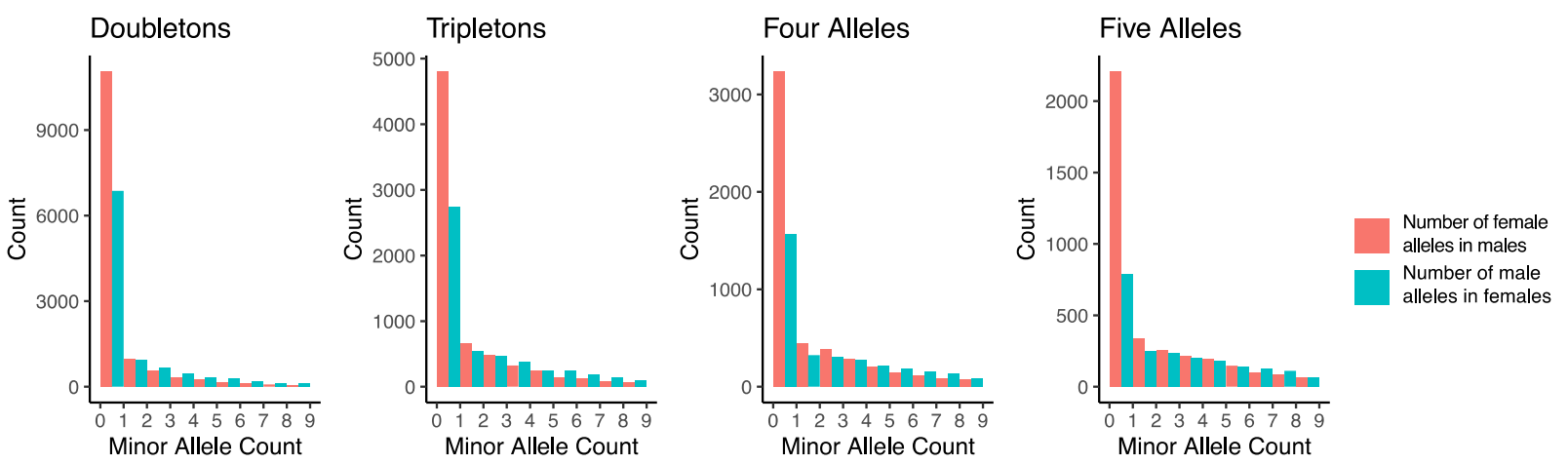

443 Figure 3: Comparison of rare nonsense and missense variants in one sex with the minor allele count in the 444 opposite sex. Red shows the distribution minor allele count of female alleles in the male dataset. Blue 445 shows the distribution minor allele count of male alleles in the female dataset. The red bar is greater than 446 in blue bar for a minor allele count of zero indicating that variants with 2-5 copies in females are more 447 often missing in males than variants with 2-5 copies in males are missing in females. 

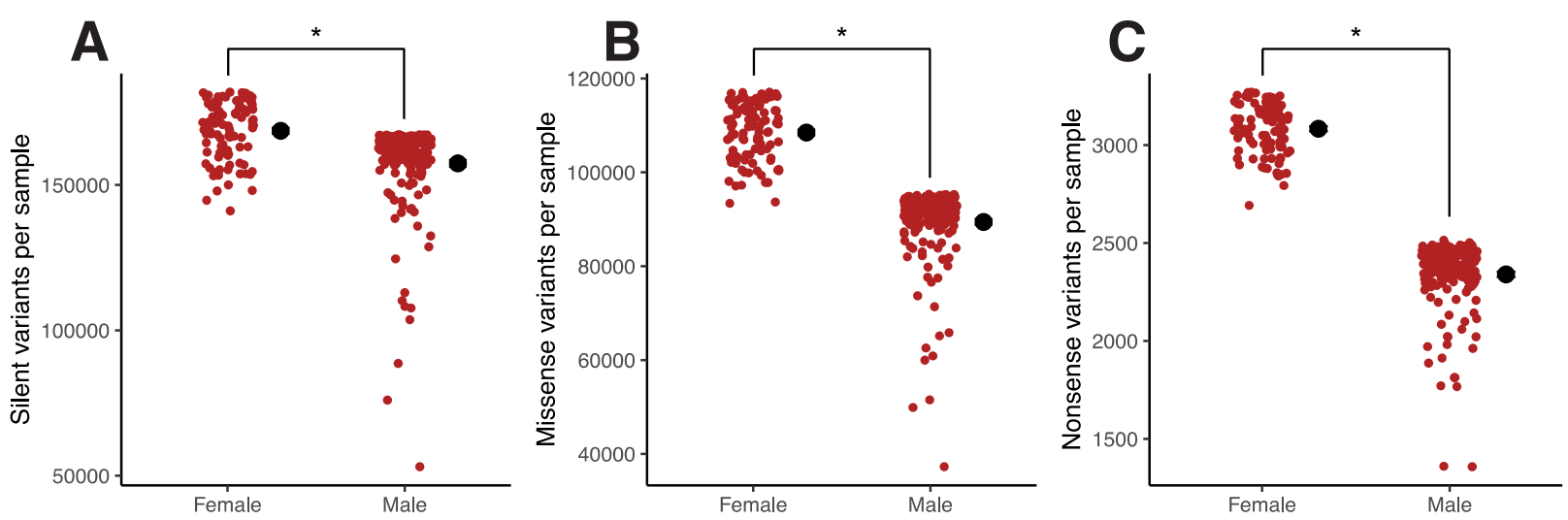

Figure 4: Genetic load lower in males. Using SnpEff, we calculated the number of (A) silent variants, (B) 451 Missense variants, and $(C)$ nonsense variants for each individual in our dataset. 
452 Table 1: SnpEff results for males and females

\begin{tabular}{|c|c|c|c|c|}
\hline \multirow[b]{2}{*}{ SNPS } & \multicolumn{2}{|c|}{ FEMALES } & \multicolumn{2}{|c|}{ MALES } \\
\hline & Total Variants & $4,784,723$ & Total Variants & $3,711,776$ \\
\hline & Genic Variants & 302,883 & Genic Variants & 266,272 \\
\hline & Missense Variants & 117,439 (38.77\%) & Missense Variants & $96,000(36.05 \%)$ \\
\hline & Nonsense Variants & $3,294(1.09 \%)$ & Nonsense Variants & $2,537(0.95 \%)$ \\
\hline & Silent Variants & $182,150(60.14 \%)$ & Silent Variants & $167,735(62.99 \%)$ \\
\hline & Missense/Silent Ratio & 0.6447 & Missense/Silent Ratio & 0.5723 \\
\hline & Ts/Tv Ratio & 1.9015 & Ts/Tv Ratio & 2.0637 \\
\hline IN/DELS & Total Variants & $1,743,723$ & Total Variants & $1,344,848$ \\
\hline & Insertions & 869,193 & Insertions & 685,520 \\
\hline & Deletions & 874,530 & Deletions & 659,328 \\
\hline & High Impact & $7,104(0.29 \%)$ & High Impact & $4,664(0.25 \%)$ \\
\hline & Moderate Impact & $4,776(0.19 \%)$ & Moderate Impact & $4,002(0.22 \%)$ \\
\hline & Low Impact & $5,129(0.21 \%)$ & Low Impact & $2,822(1.5 \%)$ \\
\hline
\end{tabular}

